\title{
New predictive value of optical coherence tomography analysis in the diagnosis of idiopathic intracranial hypertension
}

\author{
Sajjadi F, Khoshnevisan MH, b,c Doane JF, Sajjadi He,f,g
}

alnternal Medicine Resident, Jersey City Medical Center. 355 Grand St., Jersey City, USA.

bDirector, Preventive Dentistry Research Center, Research Institute of Dental Sciences, School of Dentistry, Shahid Beheshti University of Medical Sciences (SBMU).

Tehran, Iran.

'Director, WHO-Collaborating Center for Training and Research in Dental Public Health (SBMU), Tehran, Iran.

dDiscover Vision Centers, Kansas City Kansas USA.

eDirector, San Jose Eye and Laser Medical Center, San Jose, California, USA.

fDirector, Department of Ophthalmology, Acacia Medical Center. Dubai, UAE.

פDirector, Department of Ophthalmology, Iranian Red Crescent Hospital, Dubai, UAE.

Correspondence to H. Sajjadi (email: hsajjadi@yahoo.com).

(Submitted: 22 January 2017 - Revised version received: 18 February 2017 - Accepted: 25 February 2017 - Published online: 26 June 2017)

\begin{abstract}
Objective Idiopathic Intracranial Hypertension (IIH) was first reported in 1897 as "Pseudo tumor Cerebri" when lumbar puncture was initiated in medicine. However, it is still a challenging diagnosis for many neurologists and ophthalmologists. Likewise, the sequela of IIH can be debilitating overall health and quality of life in most patients. This report presents the evolving diagnostic modalities that are different from mainstream IIH reports on gender, obesity and headaches ratios. We believe, using standard optical coherence tomography $(\mathrm{OCT})$ can allow for a much earlier and more accurate diagnosis of IIH, with marked reduction in visual loss morbidity.

Methods Retrospective, observational method was used to review 171 patients' charts with suspected IIH over the past 12 years. The OCT of optic nerve and Macula was conducted by considering a list of signs and symptoms. Brain MRI, lumbar puncture, and our standard laboratory workups for optic neuropathies and visual fields were done. Visual Evoked Potentials were performed by neurologists. For purpose of this paper all IIH diagnosis fit Dandy diagnosis criteria of pseudo tumor cerebri as having a normal MRI.

Results We evaluated 164 cases that had LPs done due to our proposed OCT Patterns to rule out IIH with no bio-microscopic evidence of overt papilledema. The majority of LP positive patients had some degree of visual field damage. Twenty out of 164 cases had false positive OCTs (with negative LPS) 88\% accuracy in OCT diagnosis of IIH. No false positive OCT in Pattern-I.

Conclusion Four different OCT diagnostic Patterns presented for IIH cases. The demographics of the patients considered having $\mathrm{IH}$ by OCT Patterns I-IV and proven by positive LP; do not fit the classic demographic patterns. Classically, IIH has been reported to be observed in obese females with headaches and visible papilledema. Our findings put this classic set of presenting signs into question.

Keywords pseudo-tumor cerebri, IIH, BIH, OCT, MRI
\end{abstract}

\section{Introduction}

Idiopathic intracranial hypertension (IIH), was initially known as Pseudo-tumour Cerebri and later benign intracranial hypertension (BIH) and finally changed to IIH; is a syndrome characterized by increased intracranial pressure (ICP) of cerebrospinal fluid due to unidentified reasons. The pathophysiology of IIH is well known. ${ }^{1}$ Since we did find many associated diseases (explained later) that may be causative, we recommend going back to the originally described "Pseudo-tumour Cerebri" nomenclature since this disease is certainly not benign and it may not be idiopathic at least in a number of cases. We found that all "non-medical-field job related" patients, and most non-ophthalmologist/ neurologists also understand the term "Pseudo-tumour Cerebri" much easier and faster than IIH. Even with adequate explanation a number of patients misinterpret IIH for blood pressure and or eye pressure. We also had several patients that did not present our referral letter to neurologists and instead communicated that they had glaucoma. We, therefore, reverted to the original term of "pseudotumour Cerebri" by explaining it as a condition that acts like a tumour on the brain except that there is no tumour. After this, we did not have any communication error. Thus we strongly recommend that all IIH terminology be back to the original taxonomy: "pseudo-tumour Cerebri (PTC)".
Patients may have variable clinical presentation, which can be a challenge for timely diagnosis and treatment. A delay in diagnosis can result in irreversible optic nerve damage in the acute to sub-acute and chronic phases. PTC can negatively affect patients' overall health and quality of life. ${ }^{2-7}$ However, if diagnosed and treated early, PTC can be self-limiting in most patients. ${ }^{6}$

Men, women and children of all ages can potentially be affected. The annual incidence of PTC has been reported to be between $0.9^{8}$ to 2 per $100,000^{9,10}$ in the general population, while the incidence in obese individuals may increase by tenfold. ${ }^{2,8,10}$ A report from Libya indicates that, the incidence for PTC was three to four times higher than what has been reported from the United States. ${ }^{11}$ Our findings multiply the incidence by over a 100 .

PTC typically is observed in otherwise healthy individuals. Although, the aetiology of PTC is not well known, some factors with different degrees of association have been reported; such as, age, gender, race, body mass index (BMI), and systemic diseases; including endocrine and metabolic diseases, medications, vitamins, and exposure to heavy metals (lead). ${ }^{12}$ Some of them may be the cause of this condition, we suggest.

The ophthalmic morbidity of PTC is related to vision loss from optic nerve damage or neuropathy. ${ }^{13}$ If untreated, longstanding pressure from inside the brain, by putting pressure 
on the optic nerves commonly results in irreversible optic neuropathy with visual field loss, colour desaturation or even total loss of colour vision. ${ }^{14,15}$ Although rare, central visual acuity loss can also occur, especially in younger patients. While, with appropriate early diagnosis and treatment of PTC, the prognosis is usually excellent. The treatments in most cases are also especially easy. Therefore, we have an easily treated disease that has a very high morbidity, which makes its early diagnosis of utmost medical importance and necessity.

In patients with the classic PTC presentation of headache, obesity and overt papilledema, the diagnosis is not usually missed. But, patients who do not fit the established criteria for PTC diagnosis can suffer from visual loss that goes undetected. These patients may include males and females who are not obese or do not have headache.

The treatment of PTC is mainly composed of drug therapy, CSF shunting and optic nerve fenestration. Acetazolamide is the main drug used. ${ }^{16}$ Studies have shown that, the effect of this drug was independent of weight loss. ${ }^{17}$ The cerebrospinal fluid shunting is mainly lumbo-peritoneal. ${ }^{18}$ Optic nerve fenestration procedure is still in debate as far as its long term effectiveness in preventing optic nerve damage, but more importantly optic nerve sheath fenestration does not have any effect on the increased ICP's other sequela such as significantly reducing headaches, vertigo, dizziness, tinnitus and possible development of reflex sympathetic dystrophy or vein occlusions (explained later on). Acetazolamide appears to be the most effective agent for lowering ICP. Weight loss by itself also decreases sign and symptoms of PTC.

\section{Methods}

Patients suspected for PTC and subsequently tested with optic nerve head OCT, included patients with headache and/or dizziness and/or vertigo and/or syncope. They also included patients presenting with suspicious optic nerve head characteristics including possible pseudo-papilledema and or optic nerve drusens), optic atrophy, previous diagnosis of Low Tension Glaucoma, or diagnosis of unknown optic neuropathies even in absence of headaches or obesity. We excluded optic neuritis patients of all types by not including any patients with unilateral loss of vision with ceco-central or central visual field loss or altitudinal defects and a normal contra-lateral visual field. They were worked up and treated with accepted protocols for optic neuritis. A total of 171 patients with suspected PTC were identified. Normal range of CSF pressure in adults (in lying and to the side position) varies in different reports for example in 2 separate articles; $90-180 \mathrm{~mm}-\mathrm{H}_{2} \mathrm{O}^{18,19}$ is normal and in another around $135-200 \mathrm{~mm}-\mathrm{H}_{2} \mathrm{O}^{20}$ is considered normal. In children, the ICP is reported lower than adults, as $45-95 \mathrm{~mm}-\mathrm{H}_{2} \mathrm{O}$ and in infants the lowest or $21-80 \mathrm{~mm}-\mathrm{H}_{2} \mathrm{O}^{21}$ OCT evaluation was followed by our standard laboratory workups (Table 1) for optic neuropathies as well as visual fields for all subjects. Positive LP was defined as an OICP (in $\mathrm{mm}-\mathrm{H}_{2} \mathrm{O}$ ) of over 190 lying to the side. The majority of LP positive patients had some degree of visual field damage. All patients had MRI of the brain to rule out any intracranial mass occupying lesion. VEPs were also performed for all cases by neurologists. VEPs were read by neurology standards. Data from each chart was extracted and entered into a database for further analyses. For purpose of this paper, all PTC diagnoses fit Dandy diagnosis criteria of pseudo tumour cerebri as

\begin{tabular}{lll}
\hline Table 1. List of standard tests for laboratory workout of PTC \\
suspected cases in this study
\end{tabular}

having a normal MRI. These methods resulted in 2 major changes and findings described in the following 2 parts.

\section{Part 1: Revision of PTC Classic Attributes}

PTC is a condition that historically has been diagnosed by a neurologist or neuro-ophthalmologists. Typically, obese women with headaches and/or suspected papilledema are referred to ophthalmology for visual field evaluation. In most cases, the role of the ophthalmologist in diagnosis and treatment has been limited to visual field analysis only. Neuro-ophthalmologists have had the same steps in patient management and diagnosis as a neurologist prior to widespread use of OCT.

The currently accepted clinical presentation for PTC patients includes: overt papilledema, female sex, obesity, and history of headaches. However, in the 12 years of our study that included 164 patients, $43 \%$ were male, $56 \%$ had no current history of headaches, $62 \%$ were not visibly overweight, $0 \%$ had overt papilledema, and only $35 \%$ had a total lack of spontaneous venous pulsations (SVP's). In our study, we would like to challenge the current diagnostic criteria of PTC with the following suggestions.

Diagnosis of papilledema in our patients was not based on its classic definition. Instead, any suspicious optic nerves which included nasal elevation, pseudo-papilledema in all patients (even associated with significant hyperopia), hypoplastic discs and cupping and or optic atrophy led to performing an OCT of the optic nerve and macula. All number of patients with Retinitis Pigmentosa or other tapedoretinal degenerations were also included because of pseudo-papilledematous or atrophic optic nerves. Then using our four distinct patterns visualized on OCT and measurement of micro-papilledema by OCT, patients were diagnosed as possible increased ICP. This finding led to the three further evaluations, namely visual fields, our optic nerve laboratory workup (Table 1) and an MRI of the brain without contrast. One exception to the lab workup was that Lyme disease, Vitamin A, and PTH workups that were cancelled by primary care doctors in insured cases in about $1 / 3$ of cases because of cost. Despite our repeated medical reports to the insurance companies, they refused to accept.

This study revealed that, pseudo-papilledema is actually a rare finding on OCT of the optic nerve. All our cases of "pseudo-papilledema" had significant micro-papilledema on optic nerve analysis, eventually showed OCT Patterns I-IV and actual micro-papilledema by OCT, existed in majority of cases. The prevalence and annual incidence of LP proven-PTC in our population over a 12 year period showed linear annual increase, challenging the published literature on this subject. Our research suggests 4 different Patterns of OCT for optic 
nerve and macular findings that will be presented in part 2 below. All patients with regular headaches and or migraine obtained our routine OCT analysis and were included even with normal appearing optic nerves.

In the present study, female to male ratio is altered from classical demographics to $57 \%$ female to $43 \%$ male ratio but not a dramatic female predominance. Over half (56\%) of our patients had neither headaches (56\%) nor visibly obese (62\%), and therefore, these typical associations should also be modified. By our results visible papilledema does not have to be present. With standard criteria, the majority of our patients would be missed, often times labelled as having normal optic nerve heads, mild nasal elevation, optic atrophy, low tension glaucoma (LTG), Leber's congenital optic atrophy, or various combinations of these conditions.

Our diagnostic model for PTC consists of a positive OCT Pattern specific to PTC without overt papilledema but, with or without micro-papilledema as measured by OCT, and without significance of absent or present SVPs, with a subsequent normal MRI, leading to an LP showing high OICP. Up to 700 microns of papilledema calculated by our formula on OCT of the optic nerve was read as normal or pseudo-papilledema.

The OCTs done on these selected patients for possible PTC were A) OCT of optic nerves, including ONFL, 3D optic nerve analysis with manual measurement of disc elevation, and $\mathrm{B}$ ) OCT of macula for detection of fluid leaking from optic nerve inside retina and ganglion cell layer (GCL) damage. The Macular wide scan for better GCL analysis where done since 2012 when the software became available to us. Prior to this perifoveal thinning was evaluated as GCL damage. As explained, our diagnostic model for PTC consists of a positive OCT Pattern specific to PTC with micro-papilledema in majority of cases and subsequent LP showing high OICP.

\section{Part 2: OCT Patterns Indicating Presence of PTC}

\section{Pattern-I (P-1)}

"Temporal wings with nasal depression". This is when a temporal increase in NFL thickness is seen with depression of nasal NFL. (Fig. 1) Visual field defects noted in this group ranged from non-specific scotomas to very non-congruous bi-temporal scotomas with nasal changes. Our theory is that, nasal nerve fibre layers get damaged early in PTC, because of decussating areas of nerve are very pressure sensitive. The Temporal wings are from CSF leaking into temporal disc although temporal to disc, are still nasal to the centre of macula. The same area of ANFL contains the temporal nerve fibre layer or nasal field. The excess fluid fails to show the damage to the temporal retina or nasal defects in this stage. But visual field analysis does exhibit some nasal defects.

Pattern-I patients had normal macular OCT structure and only a few had very mild GCL damage. Our hypothesis for this pattern is that, in acute PTC, the chiasm gets damaged first, at the same time the nasal optic nerve head can absorb a lot of CSF before leaking it into the adjacent retina so the nasal NFL damage shows on OCT as nasal depression. While the temporal wings are possibly result of more temporal leakage of fluid from optic nerve into the retina since the temporal optic nerve head has little capacity for holding CSF. Nasal depressions alone have been previously reported with migraine headaches. ${ }^{22}$ Since in our study over $60 \%$ of previously-diagnosed migraine headaches turned out to be PTC, the mentioned article's decreased nasal ANFL may have actually been PTC. After treatment, the high temporal to the nasal ratio of this Pattern is maintained, but overall decrease of ANFL occurs due to decreased papilledema. After decreased papilledema resultant, decreased CSF in the retina, ANFL analysis starts matching the visual field defects (Fig. 1C and D). Similar to our treated PTC patterns (Fig. B), this pattern has been seen previously ${ }^{23}$ in treated severe $P T C$ with papilledema. If $P T C$ is undetected and untreated, Pattern-I can progress to Pattern-II.

\section{Pattern-II (P-2)}

In this pattern (Fig. 2) temporal wings continue but decreased nasal ANDL is now increased, along with both increased superior and or inferior increase is seen in more chronic PTC. All patients exhibit visual field defects of various types like Pattern-I. Our hypothesis for this Pattern is that there is leakage of CSF in the nasal engorged optic disc into the retina, thus masking the nasal damage and showing a nasal increase in the nerve fibre layer. Majority of these cases also had increased superior and inferior peaks of the nerve fibre layer. In contrast in glaucoma the superior and inferior NFL is usually thinned early on and correlates with the VF loss. So this is a separating point in PTC and glaucomatous damage.

\section{Pattern-III (P-3)}

In this Pattern the nasal elevation persists while the temporal optic atrophy show as depressed temporal ANFL (Fig. 3). So VFs in this stage had both bi-nasal and bi-temporal defects while still saving large central field area. "Nasal humps" in the presence of normal or elevated inferior and temporal NFL and with temporal depression. This is seen usually in more chronic PTC which typically shows moderate visual field defects, but lacking deep central scotomas. In contrast to glaucoma where there are more central scotomas. The amount of GCL damage was less than similar glaucomatous average estimated field loss cases.

\section{Pattern-IV (P-4)}

"Greatly decreased nasal nerve fibre layer with normal or increased temporal nerve fibre layer (NFL)" is seen in much older patients with decades of PTC (Fig. 4). This is also in contrast to glaucoma, whereas disease progresses, one preserves more nasal than temporal NFL, and thus the temporal field remains in late stage glaucoma (nasal nerve fibre layers). All patients had less than 20/50 visual acuity but none had tunnel vision complaints (except combined with Retinitis Pigmentosa). Several patients had below legally blind visions of 0.2 to NLP. Few patients showed central scotomas, and the only differentiating factor from glaucoma was more temporal nerve fiber layers than nasal and lack of a temporal island.

\section{Results}

Number of patients in different patterns were divided as follows: Pattern-I $(n=35)$, Pattern-II $(n=58)$, Pattern-III ( $n=$ $51)$ and Pattern-IV $(n=20)$. Headache was the chief complaint in small portion (13\%) of our sample and $92(56 \%)$ patients had no history of significant headache. Non-regular headaches associated with hunger and or not requiring taking medication were considered non-significant headaches. Over half of our patients did not have headaches (56\%) nor visible obesity (62\%). However, by BMI $42 \%$ were non-overweight, which is explained later. Also, our study revealed that, 107 of 164 (65\%) of our patients, with positive LPs had visible SVP's. Thus, only $35 \%$ were recorded as nonpresent SVPs. Any movements of veins, no matter how slight 


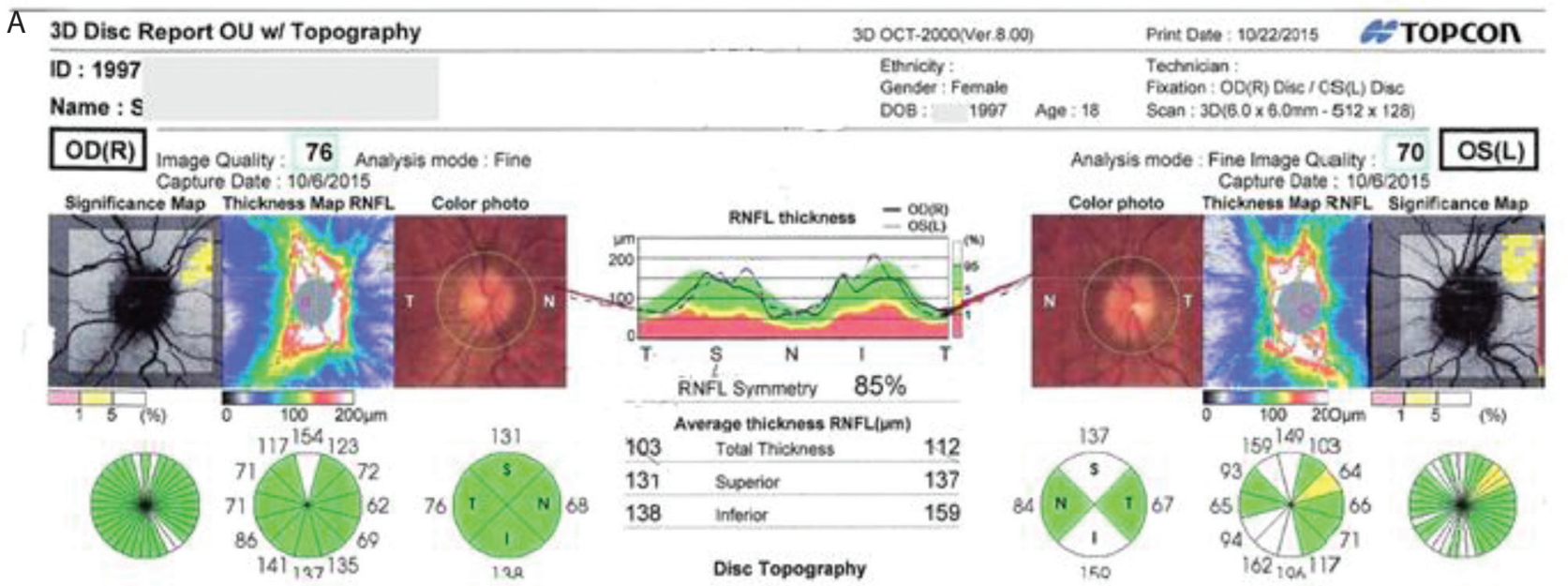

B

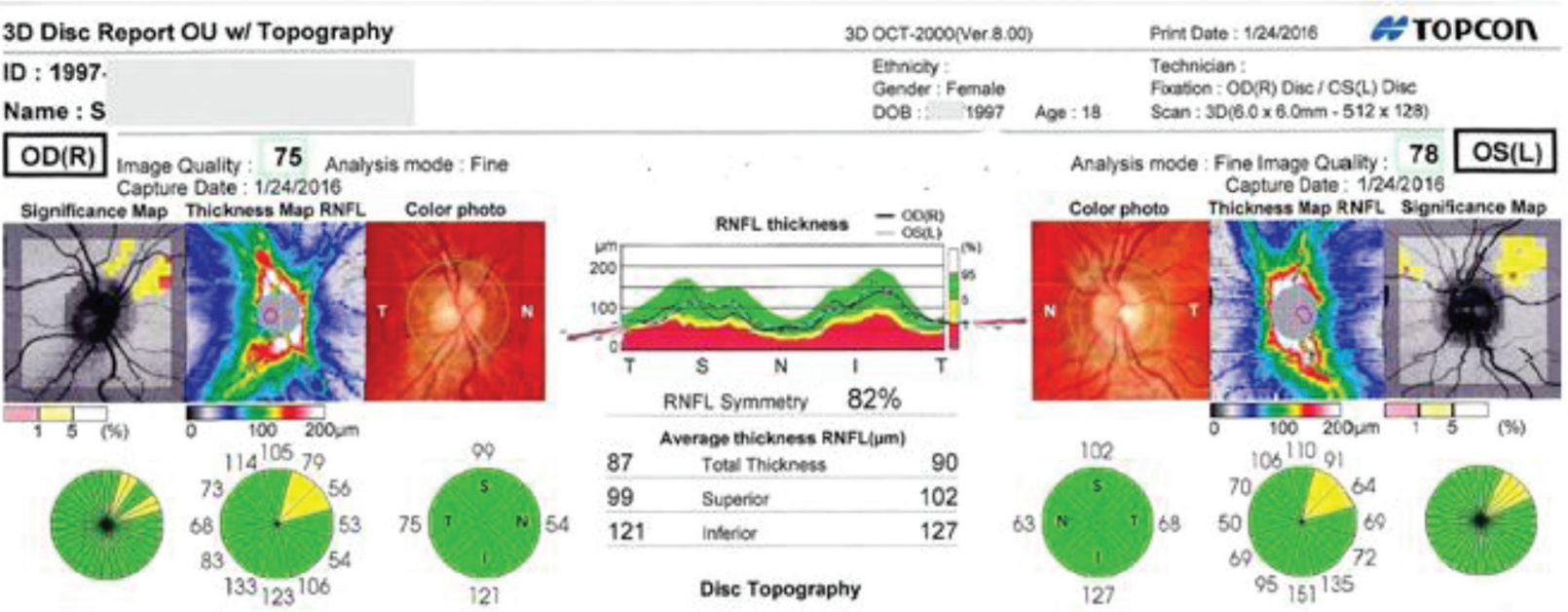

Legend: OD continuous line, OS dotted line. 18 year old female not overweight no headaches.

Fig. 1 Pattern-I (P-1): (A) Before treatment. Temporal wings with nasal depression. No overt papilledema. Optic nerve visually rated as mild nasal elevation flat temporal. Micro-papilledema measured at 1000 microns. (B) 5 months after treatment. Overall decreased ANFL, now showing superior nasal defects. But maintaining a high temporal to nasal ratio with flat temporal extensions. Optic nerve shows increased cup size and less congestion.

were considered as SVPs. If we adjust this for 20 false positive OCTs with negative LPs, this ratio corrects to $54 \%$ of $+\mathrm{LPs}$ had SVP and $46 \%$ had no SVPs. Therefore, these typical associations with papilledema should be further studied.

Out of our 171 OCT patterns referred for MRI, a total of 7 cases were excluded from the study because 4 had overt papilledema and 3 had "papilledema and brain tumours" on MRI. All the remaining 164 of the 171 cases had LPs done and the opening OICP were in the range of $180-600 \mathrm{~mm}-\mathrm{H}_{2} \mathrm{O}$. Eight (5\%) cases had LP-OICP of $180-210 \mathrm{~mm}-\mathrm{H}_{2} \mathrm{O}$. In 141 $(86 \%)$ cases the results were between 220 to $350 \mathrm{~mm}-\mathrm{H}_{2} \mathrm{O}$. And 15 (9\%) of cases had LP-OICP of $>360 \mathrm{~mm}-\mathrm{H}_{2} \mathrm{O}$.

We observed positive OCT changes but borderline OICP of $180-210 \mathrm{~mm} \mathrm{H}_{2} \mathrm{O}$ in 8 patients. Therefore, these cases were considered true PTC, because treatment with Diamox resulted in stop of visual field loss and decrease in ANFL thickness. In patients with headaches and obesity it also resulted in significant loss of headaches and or loss of weight.

In our cohort, there were $71(43.3 \%)$ male and 93 (56.7\%) female patients. The age range of patients was 4-79 years. Thirty three cases (20\%) were over age 55 and 97 cases (59\%) were between $26-55$ years of age and 34 (21\%) were under age 25. The racial trends in our study included 41 African cases (25\%), 49 cases (30\%) Caucasian Western European Origin or descent, and 74 cases (45\%) of Caucasian Middle-Eastern origin or descent. One hundred and forty (85\%) patients were residents of the Middle Eastern countries.

Out of the 164 patients only 21 (13\%) had headaches as chief complaint. Although 51 cases (31\%) did not initially volunteer a history of headache. However, due to our extensive questioning about signs of headaches in the past, these 51 patients revealed a history of headache and/or migraine within many years prior that had completely gone away. A total of 92 (56\%) patients had no history of headache at all, which is a substantial finding. These patients were suspected of increased ICP and worked up with OCT, due to suspicious reasons that have already been explained.

Of the 7 excluded cases 4 had typical PTC OCT pattern (Fig. 5), which has been reported before, but this pattern is also shared with other optic neuropathies. All 4 patients had overt papilledema. Of these, one had normal MRI and 3 had Chiari-type-I MRI diagnosis and they had LP opening 


\begin{tabular}{lcl} 
3D Disc Report w/ Topography & 3D OCT-2000(Ver.8.00) & Print Date : 12/18/2014 $\mathcal{C}$ TOPCON \\
\hline ID : & Ethnicity : & Technician: \\
Nam & Gender : Female & Fication : OD(R) Disc \\
DOB : $\quad$ & Age : 5 & Scan : $3 D(6.0 \times 6.0 \mathrm{~mm}-512 \times 128)$ \\
\hline
\end{tabular}

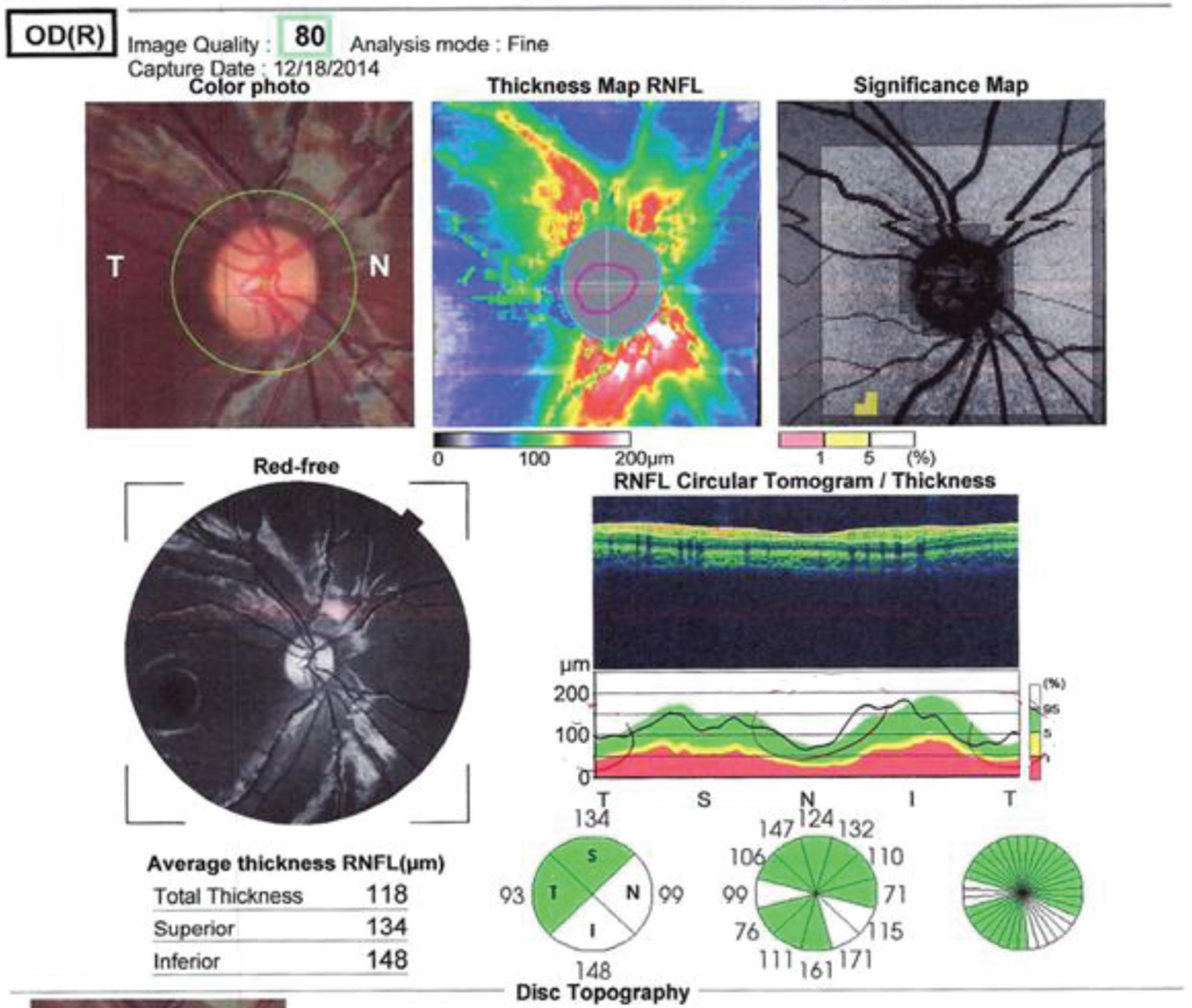

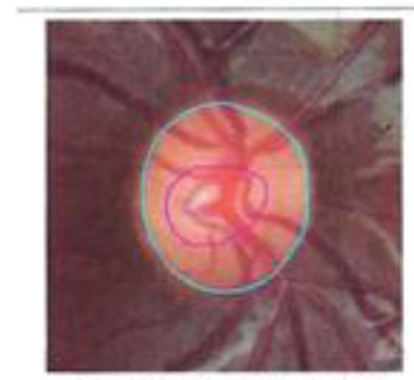

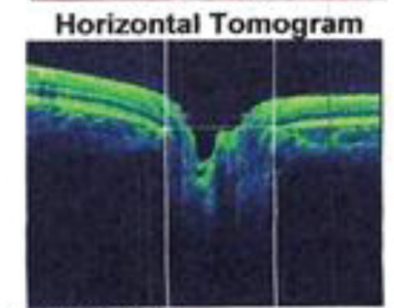

Comments :

Iranian Hospital

\begin{tabular}{lll} 
Disc Area & $\left(\mathrm{mm}^{2}\right)$ & 3.14 \\
\hline Cup Area & $\left(\mathrm{mm}^{2}\right)$ & 0.79 \\
\hline Rim Area & $\left(\mathrm{mm}^{2}\right)$ & 2.35 \\
\hline C/D Area Ratio & & 0.25 \\
\hline Linear CDR & & 0.50 \\
\hline Vertical CDR & & 0.44 \\
\hline Cup Volume & $\left(\mathrm{mm}^{3}\right)$ & 0.13 \\
\hline Rim Volume & $\left(\mathrm{mm}^{3}\right)$ & 0.09 \\
\hline Horizontal D.D & $(\mathrm{mm})$ & 1.84 \\
\hline Vortical D.D & $(\mathrm{mm})$ & 2.16 \\
\hline
\end{tabular}

Disc margin — Disc parameters are determined at the reference plane height of 120

Cup margin -

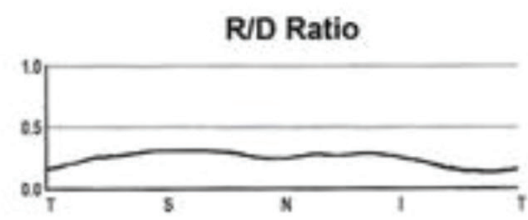

um from the RPE plane in this version.

Fig. 2 Pattern-II (P-2), OD 4 year old girl with Amblyopia, high hyperopia and no overt papilledema. Temporal wings with increased naso-inferior NFL in both eyes and LP positive. 


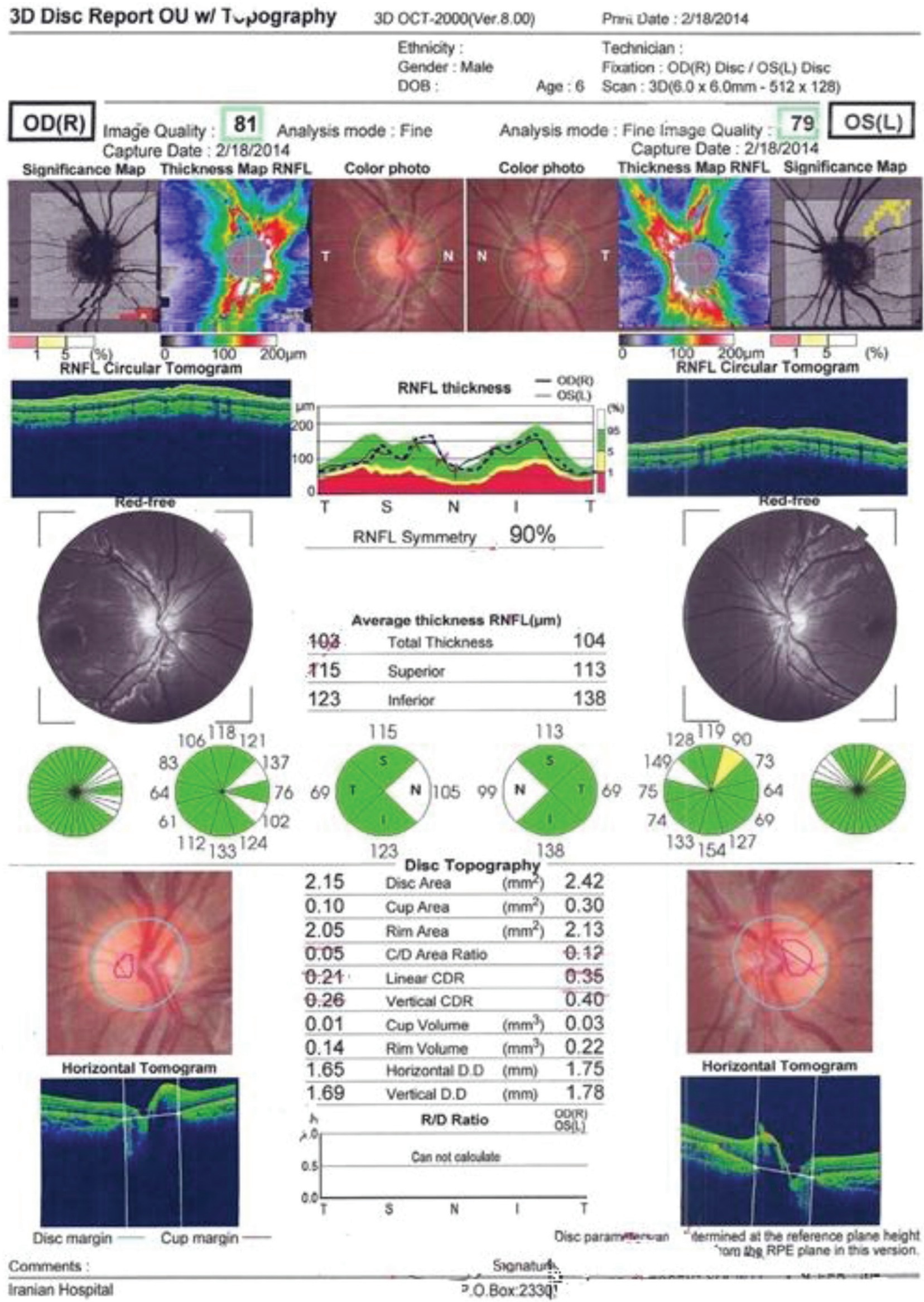

Fig. 3 Pattern-III (P-3) 6 year old female pseudo-papilledema, hyperopic, not overweight and no headaches. Nasal and inferior elevation with temporal decrease in ANFL and LP positive. 


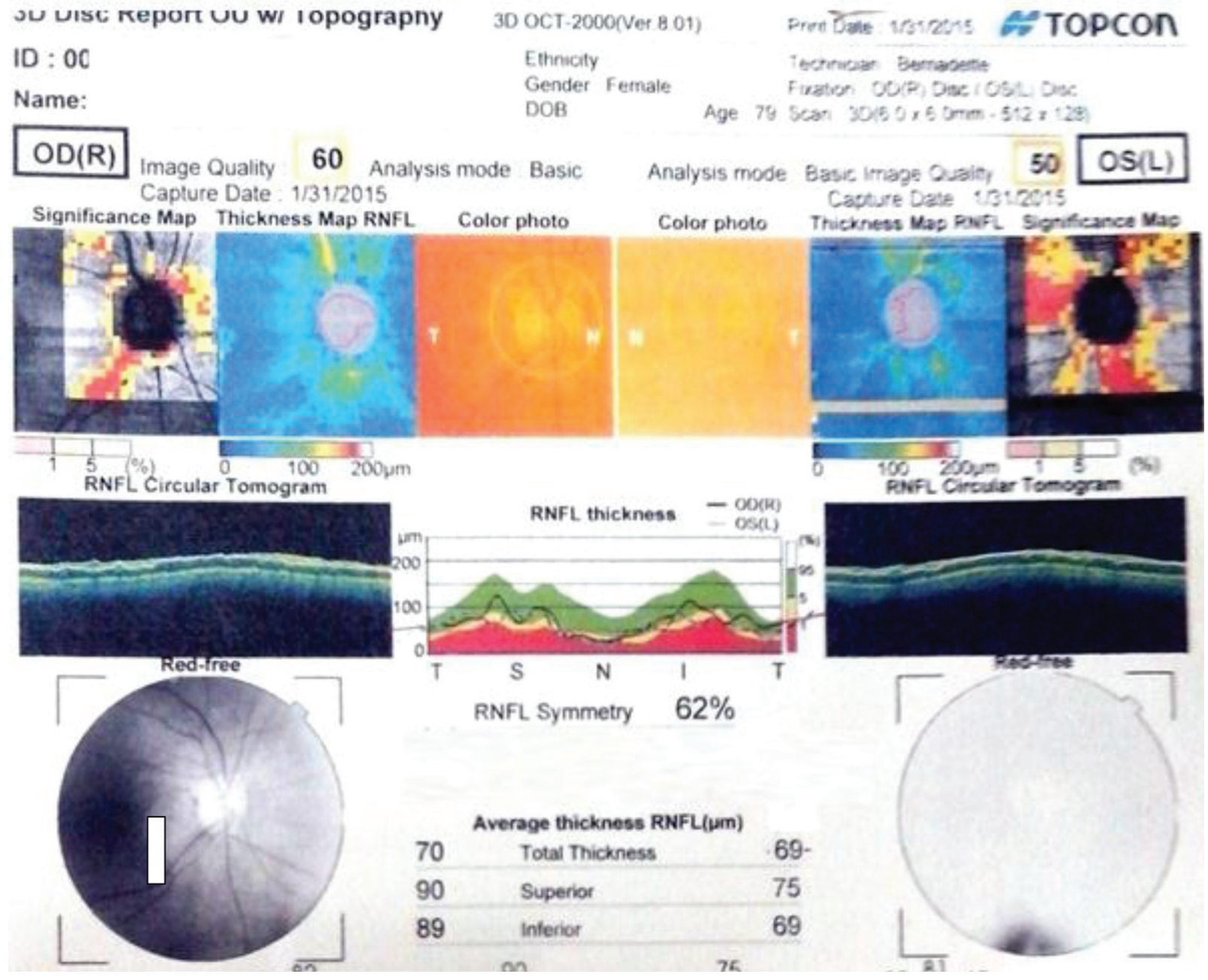

Fig. 4 Pattern-IV (P-4) 79 years old male overweight no headaches with previous diagnosis of LTG on triple drops. Pattern 4 shows overall decrease in nerve fiber layer but still more so nasally than temporally. This patient had an ICP of 300. IOP on triple drops 17/17. Now on Acetazolamide 250 QID; off all drops; IOP 18/18.

pressures over $300 \mathrm{~mm}-\mathrm{H}_{2} \mathrm{O}$. It is important to mention that, this previously reported across the line increase in ANFL may be seen without papilledema, and still be indicative of PTC.

The other 3 excluded patients were female and had pituitary tumours on MRI and therefore, were referred for neurosurgical evaluation. Each of these patients had very high Prolactin levels and thus Prolactin releasing adenomas. ${ }^{20}$ Mild to moderately elevated Prolactin levels was also seen in pure PTC.

The highest number of cases in our sample demonstrated Pattern-II $(n=58)$, followed by Pattern-III $(n=51)$, Pattern-I $(n=35)$, and Pattern-IV $(n=20)$. Headache was the chief complaint in small portion (13\%) of our sample and 92 (56\%) patients had no history of significant headache. Non-regular headaches associated with hunger and or not requiring taking medication were considered non-significant headaches.

In Pattern-I OCT for increased ICP we had 35 (21.3\%) patients that were diagnosed with PTC with $100 \%$ positive LP. These patients showed none to mild decrease in GCL. Visual fields showed some temporal and nasal defects in a nonspecific way however nasal defect was more than temporal defect. VEPs were all normal.

Pattern-II was seen in 58 (35.4\%) patients. Only 7 patients (12\%) had a false positive OCT pattern with negative LP. All patients exhibit visual field defects of various types. We are also calling this pattern very specific of increased ICP. All patients had mild to moderate GCL damage. Visual field were similar to Pattern-I but showed more para-central scotomas.

Pattern-III was observed in 51 (31.1\%) patients. Eight patients $(16 \%)$ had a false positive OCT pattern with negative LP. Our hypothesis for this pattern is that, the temporal nerve fibre layer is now showing its damage and leakage of CSF of the nasally engorged optic disc into juxta-papillary nasal retina occurs where the OCT circle, measures ANFL, thus masking the nasal damage. Although visual fields remained non-specific, they all had a hint of bi-temporal defects with some typical glaucoma like para-central and nasal visual field damage.

Pattern-IV was seen in 20 or $12.2 \%$ of patients. Five of these 20 patients (25\%) demonstrated false positive OCT pattern with negative LP. So this pattern was $75 \%$ specific. All of these patients showed macular thinning and mainly moderate to severe GCL damage. Our hypothesis for Pattern-IV is that, at this stage, the optic nerve has atrophied; the nasal nerve fiber layer is mostly damaged, thus shifting the CSF more temporal into the retina masking the temporal damage. The temporal ANFL is also damaged, but because of very low capacity of temporal optic nerve head, there is mild leakage of CSF, thus the temporal NFL seems normal. 


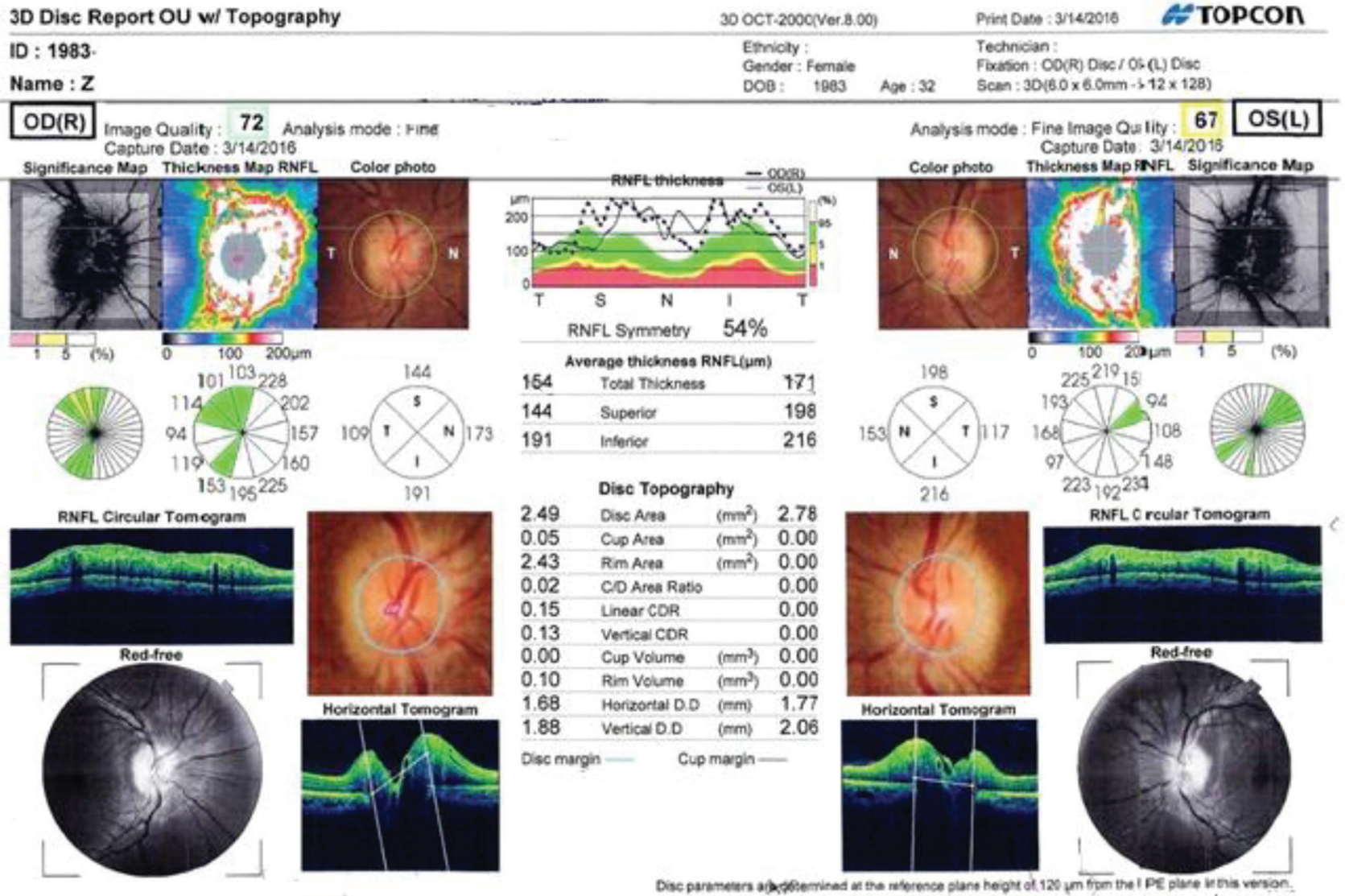

Fig. 5 Typical increased ICP pattern OCT, with overt papilledema, and ANFL 2x normal. A 32 year old lady referred by another ophthalmologist with diagnosis of severe papilledema. Papilledema measured on OCT 1000-1500 microns 0U.

This is in contrast to advanced glaucoma when you almost always have more temporal NFL damage seen on OCT since there is no fluid leaking into the retina in glaucoma and nasal steps correspond to temporal NFL damage. Some of these patients were diagnosed as glaucoma prior to our diagnosis, but lack of temporal NFL damage should alarm one that, this may not be glaucomatous change. Majority of this group had dense visual field defects with some central scotomas, but a larger temporal than nasal visual field damage was the rule.

Majority of OCT Patterns 1 to 3 patients did not exhibit VEP defects, while all patients with Pattern 4, showed VEP defects since OCT and VF estimate of ANFL loss exceeded $40 \%$. Therefore, VEP does not seem to be a good diagnostic tool for mild to moderate PTC since the lateral geniculate body magnifies the signal so much, that there is no change in VEP unless there were $40 \%$ ANFL and or VF loss.

\section{Comparing OCT Patterns I-IV with Glaucoma Patients}

Early glaucoma patients have superior and or temporal depression initially of the ANFL. Although, advanced glaucoma demonstration is usually opposite to our Pattern-III, showing continued superior and temporal depression with some nasal depression and no temporal wings. In macular OCT examination most glaucoma cases show some GCL damage; while moderate to advanced glaucoma shows moderate to advanced GCL damage. Therefore, in differential diagnosis, macular thinning (GCL damage) and optic nerve damage have a linear response in glaucoma and not as much in PTC. The reason for this may be that, in glaucoma the GCL is damaged early by eye pressure, while in PTC the GCL damage is retro-grade from brain pressure. The younger the patient, presumably with higher OICPs, the more acute decreased nasal nerve fiber layers with double or tripled temporal ANFL thickness that we labelled as "temporal wings with nasal depression". (Fig. 1)

Based on LP proven PTC, it is our assumption that, the reason for remission in previously diagnosed headaches between ages 30-40 is secondary to the brain getting used to the high ICP and thus not triggering a vascular response. In other words, the PTC patients with headache behave same as classic migraine in remission after 10-15 years of severe headaches, while their ICP stays high and progressive but very slow damage to optic nerve continues.

Of the Seventy two (44\%) patients in our study with current or previously diagnosed headaches by neurologist and primary physicians; 50 (69\%) displayed our specific OCT Patterns, and had positive LPs.

Majority of overweight patients in our study lost weight on oral acetazolamide. Remarkably, all the patients who had history of relief of headaches on Topiramate prior to our PTC diagnosis had positive LPs. This leads us to suggest to neurologists that all headache patients responding well to Topiramate and especially with loss of weight should be worked up for PTC.

\section{Discussion}

In our study, we would like to show a similar yet different association of OCT to the visual field. Although, our OCT Patterns 
are extremely specific for $P T C$, these are usually read as normal or at best glaucomatous by the majority of ophthalmologist. This is mainly due to the fact that, in early cases the visual fields were either normal or have non-specific patterns, such as a few temporal and nasal defects. Visual fields show more of a bi-temporal filed loss than nasal in more advanced cases. Therefore, our main focus was to detect PTC conditions as early as possible with OCT then follow relying more on visual fields. Treated cases were followed up with OCT of the optic nerve, GCL map and visual fields.

The VEPs in our cases did not show any change until there was at least a $40 \%$ ANFL loss by either OCT or VF loss estimates. We mention estimates, since our proposed OCT Patterns; although diagnostic do not show the actual visual field damage early on and before treatment. We estimated ANFL loss by apparent scotomas in the visual field and by a formula we have developed, which is comprised of decreasing the ANFL in relation to micro-papilledema. Alarmingly in our study, in patients less than 30 years old, there were cases of sudden blindness or severe and rapid loss of vision. However, in patients older than 30 years of age, it tends to take a much longer time for optic nerve damage to occur in PTC.

We observed 4 OCT Patterns in our patients, which did not have overt papilledema. Typical PTC OCTs with overt papilledema, show generalized, and very high increase in ANFL (over 130 microns) which have been previously reported (23). All the acute optic neuritis cases that were excluded from this study also showed this pattern of ANFL. In the differential diagnosis of highly acute PTC, with overt papilledema, optic neuritis must be ruled out, although several initially misdiagnosed and treated with high dose steroids were not hurt but later continued on Acetazolamide instead of steroids. In our experience the optic neuritis was usually unilateral and had extensive GCL damage with central visual field loss, while acute overt PTC associated papilledema did not have much GCL damage nor central visual field loss.

Using our criteria for performing OCT; overall ANFL was increased to 105-135 micron level in Patterns I-II. Patients with Pattern III had a range of $80-125$. The patients with Pattern-IV however, had a low or normal range of ANFL 48-85 microns. As previously stated, since 2003 and over thousands of OCTs we have refined our normal ANFL to 85-110 microns. Some of these patterns have been seen in glaucoma or other demyelinating diseases in the past. To clearly see these patterns we have extended the temporal nerve fiber layer by hand in the same angular direction to produce what we call temporal wings (TWs). The higher these temporal wings on either or both temporal NFL, the higher chance of positive LPs thus PTC where found.

As mentioned we had no false positive OCT in Pattern I since all OICPs were over 190. However, all the false positive OCTs in Pattern II had OICPs of 170-180 and some of these patients were treated if they had headaches and or obesity. In Pattern III and IV the low OICPs ranged 140-180 and some of these patients were also treated as above.

In maculopathies such as macular pucker or CME, we have seen what we call pseudo-temporal wings because of reading of elevated internal limiting membrane. None of our patients have these non-CSF induced (CSFI) pathological maculopathies that would induce a false temporal elevation of the ANFL.
Interestingly, we had 6 (4\%) patients with CSF leakage from $\mathrm{ONH}$ to the macula causing CME (CSFI-CME) and thus true and very elevated TWs (Temporal Wings). One of these patients with severe optic atrophy and our Pattern-IV OCT LP-OICP of $350 \mathrm{~mm} \mathrm{H}$ O, was a 55 year old man with previous diagnosis of LTG and CME on triple drops for glaucoma. He had undergone lensectomy OU, because of decreased vision with no improvement in VA 6 years prior and had 2 intra-vitreous injections of both steroid and antiVGEF with no improvement for presumed post cataract CME. This patient's CSFI-CME improved after Acetazolamide therapy, but his visual acuity, optic atrophy, and visual fields, did not improve. These signs have remained stable after 2 years on oral therapy and discontinuation of his glaucoma eye drops, but the PTC induced TWs were decreased after treatment. It should be obvious that the ANFL in all cases of PTC decrease after treatment.

Five of the above 6 patients were diagnosed as CSFI-CME by us, without any CME treatment, which resolved after Acetazolamide therapy. In one pseudophakic patient who had improved but persistent CME, suggesting that he may have had both pseudophakic CME and CSFI-CME. Therefore, patients being considered for CME or optic nerve pit related macular fluid should also have an OCT of the optic nerve of both eyes to rule out papilledema or CSF induced macular edema (CSFI-CME).

This finding of temporal wings becomes a problem in diagnosis of patients with macular pucker and headaches since the temporal wings may be from the pucker. In these cases other parts of OCT and visual filed should be used for possible MRI indication.

Our overall false-positive OCTs with negative LPs were 20 out of 164 or $12 \%$. In our cases presence of true "temporal wings" with nasal depression (Pattern-I) can be called pathognomonic of PTC.

Objectively for reporting it would be advised to use body mass index as the de facto reporting measure. In our 144 patients with LP proven PTC, 55 (38\%) were described overweight or obese by observation. Therefore, $62 \%$ were not described as overweight.

After 2013 we started using BMIs in patients. After that $43 \%$ of cases had BMIs of 25-30 who were not visibly overweight. Fifteen percent of patients had BMI of over 35 or were severely obese. By using BMI after 2013, 58\% of our patients were overweight to obese. Thirty one percent of patients had normal BMIs of 19-25. 12\% of patients BMIs less than 19. All of these patients were under age 25 and majority were male some as young as 4 years old. Therefore, not having obesity or being undernourished does not rule out PTC.

As the majority of ophthalmologists like us at least before Electronic Health Records (EHR), did not calculate a BMI, they often did not consider PTC in a visibly non-obese patient, unless they had overt papilledema. This may count for lower incidence of PTC in the past.

Some undiagnosed patients may suffer from persistent significant headaches, dizziness or vertigo, tinnitus, depression, anxiety and insomnia. ${ }^{24}$ These patients may exhibit slowly progressive visual loss or even blindness. ${ }^{25}$ The relapse rate of PTC symptoms after treatment and gradual taper and or cessation of therapy has been reported at $28 \%$ after 2 years, ${ }^{6} 38.4 \%$ after 6.2 years $^{26}$ and $15 \%$ after 10 years. ${ }^{27}$ Our relapse rates are not subject of this study. Although rare, 
rapid central visual acuity loss can also occur. ${ }^{28}$ We have particularly seen acute and sub-acute extensive visual loss in patients under the age of 30, making their rapid diagnosis "of utmost importance."

In light of our OCT findings and the near direct correlation to $P T C$ we would recommend consideration that, headache and migraine patients requiring multiple physician visits for the headache at any age or any BMI, should be considered for an OCT of optic nerve and macula, and if they revealed one of our 4 "OCT Patterns" mentioned here, they should have an MRI and if normal, undergo an LP as well.

Majority of previous PTC articles considered 180-240 $\mathrm{mm} \mathrm{H}_{2} \mathrm{O}$ as suspicious and pressures over 250 as truly PTC. ${ }^{29}$ We believe if patients show our OCT Patterns I-II have PTC no matter what the OICP since there are also reports of PTC with low OICP. ${ }^{30}$

When patients in addition to having our previously explained reasons for getting an OCT of ON and Macula and then MRI and LP had any of the following associated diseases, there were absolutely no negative LPs. These illnesses were central or branch retinal vein occlusion, Tapedo-retinal degenerations, optic disc hypoplasia, high vitamin A levels (without history of being on vitamin A), combined "Vitamin B12 and vitamin D" deficiencies, Lyme Disease, Sleep apnea, Endometriosis, Crohn's disease, Parathyroid abnormalities (not associated with low vitamin D), and Peripheral Sympathetic Dystrophy. Thus, we recommend that any patients with above illnesses be referred for an OCT of the optic nerve and macular GCL by the respected subspecialties, such as rheumatology. And likewise any patient suspected of unexplained optic neuropathy should be questioned or evaluated for these conditions. We are analysing the data on all these associated diseases and plan a separate publication for their percentages and results of Acetazolamide therapy on their outcome.

Therefore, our findings are putting all these classical signs and symptoms of PTC under question. Visible Papilledema or overt papilledema with no spontaneous venous pulsations (SVPs) is not a major point in this study. We present evidence of micropapilledema as measured with OCT and typical Patterns that are extremely specific for PTC. Our criteria revealed that 107 of 164 (65\%) of our patients, had micro-papilledema with visible SVPs. Thus, only $35 \%$ were recorded as non-present SVPs.

\section{Conclusion}

We must take into consideration that, the quality of life for patients and their families is negatively affected due to PTC disorder. Patients may lose vision due to misdiagnosis or delayed diagnosis at early stages and/or improper treatment of PTC cases. It seems conventional criteria should be revisited based on these new clinical findings. A multicenter prospective study to see if our suggested Patterns; especially P-1 and P-2, are as precise as we found must be considered.

\section{Conflict of Interest}

None.

\section{References}

1. Johanson CE, Duncan JA $3^{\text {rd }}$, Klinge PM, Brinker T, Stopa EG, Silverberg GD. Multiplicity of cerebrospinal fluid functions: new challenges in health and disease. Cerebrospinal Fluid Res. 2008;5:10.

2. Friedman DI, Jacobson DM. Idiopathic intracranial hypertension. J Neuroop hthalmol. 2004;24:138-145.

3. Ball AK, Clarke CE. Idiopathic intracranial hypertension. Lancet Neurol. 2006:5:433-442.

4. Wall M. Idiopathic intracranial hypertension. Neurol Clin. 2010;28: 593-617.

5. Digre, KB. Idiopathic intracranial hypertension. BMJ. 2010;341:c2836.

6. Biousse V, Bruce BB, Newman NJ. Update on the pathophysiology and management of idiopathic intracranial hypertension. J Neurol Neurosurg Psychiatry. 2012;83:488-94

7. Yri HM, Wegener M, Sander B, Jensen R. Idiopathic intracranial hypertension is not benign: a long-term outcome study. J Neurol. 2012;259:886-894.

8. Durcan FJ, Corbett JJ, Wall M. The incidence of pseudotumor cerebri. Population studies in lowa and Louisiana. Arch Neurol. 1988;45:875-877.

9. Wall M. Idiopathic intracranial hypertension (Pseudotumor cerebri). Curr Neurol Neurosci Rep. 2008;8:87-93.

10. Radhakrishnan K, Ahlskog JE, Garrity JA, Kurland LT. Idiopathic intracranial hypertension. Mayo Clin Proc. 1994;69:169-180.

11. Radhakrishnan K, Thacker AK, Bohlaga NH, Maloo JC, Gerryo SE. Epidemiology of idiopathic intracranial hypertension: a prospective and case-control study. J Neurol Sci.1993;116:18-28.

12. Chen J, Wall M. Epidemiology and risk factors for idiopathic intracranial hypertension. Int Ophthalmol Clin. 2014;54:1-11.

13. Digre KB, Nakamoto BK, Warner JE, Langeberg WJ, Baggaley SK, Katz BJ. A comparison of idiopathic intracranial hypertension with and without papilledema. Headache. 2009:49:185-193.

14. Corbett JJ, Savino PJ, Thompson HS, Kansu T, Schatz NJ, Orr LS, et al. Visual loss in pseudotumor cerebri. Follow-up of 57 patients from five to 41 years

and a profile of 14 patients with permanent severe visual loss. Arch Neurol. 1982;39:461-474

15. Agostoni E, Aliprandi A. Alterations in the cerebral venous circulation as a cause of headache. Neurol Sci. 2009;30:S7-10.

16. Hughes S. Drug improves vision in idiopathic intracranial hypertension. Medscape Medical News. Available at http://www.medscape.com/ viewarticle/824073. Accessed: April 29, 2014

17. Wall M, McDermott MP, Kieburtz KD, Corbett JJ, Feldon SE, Friedman DI, et al. Effect of acetazolamide on visual function in patients with idiopathic intracranial hypertension and mild visual loss: the idiopathic intracranial hypertension treatment trial. JAMA. 2014;311:1641-1651.

18. Burris CA, Ashwood ER, Burns DE. $4^{\text {th }}$ ed. Tietz textbook of clinical chemistry and molecular diagnostics. St. Louis: Elsevier Saunders. 2006;1633:962-967.

19. Kaplan, Pesce. Clinical chemistry: theory, analysis, correlation. $5^{\text {th }}$ ed St. Louis, Ml: Elsevier Inc. 2010;904-928.

20. Mindermann T, Wilson CB. Age-related and gender-related occurrence of pituitary adenomas. Clin Endocrinol (Oxf). 1994;41:359-364.

21. Dunn LT. Raised intracranial pressure. J Neurol Neurosurg Psychiatry. 2002;73:i23-i27.

22. Rana Sorkhabi, Somaiyeh Mostafaei, Mohammad Hosein Ahoor, Mahnaz Talebi. Evaluation of retinal nerve fiber layer thickness in migraine. Iran J Neurol. 2013;12:51-55.

23. Kupersmith MJ, Kardon R, Durbin M, Horne M, Shulman J. Scanning laser polarimetry reveals status of RNFL integrity in eyes with optic nerve head swelling by OCTScanning laser polarimetry reveals RNFL integrity. Invest Ophthalmol Vis Sci. 2012;53:1962-1970.

24. Kleinschmidt JJ, Digre KB, Hanover R. Idiopathic intracranial hypertension: relationship to depression, anxiety, and quality of life. Neurology. 2000;54:319-324

25. Wall M, George D. Idiopathic intracranial hypertension. A prospective study of 50 patients. Brain. 1991;114( Pt 1A):155-180 
26. Kesler A, Hadayer A, Goldhammer Y, Almog Y, Korczyn AD. Idiopathic intracranial hypertension: risk of recurrences. Neurology. 2004;63: 1737-1739.

27. Shah VA, Kardon RH, Lee AG, Corbett JJ, Wall M. Long-term follow-up of idiopathic intracranial hypertension: the lowa experience. Neurology. 2008;70:634-640.
28. Ney JJ, Volpe NJ, Liu GT, Balcer $\sqcup$, Moster ML, Galetta SL. Functional visual loss in idiopathic intracranial hypertension. Ophthalmology. 2009;116:1808-1813.e1.

29. Diagnostic criteria for idiopathic intracranial hypertension. Friedman DI, Jacobson DM. Neurology. 2002;59:1492-1495.

30. Suh SY, Kim SJ. IIH with normal CSF pressures?. Indian J Ophthalmol. 2013:61:681-682.

This work is licensed under a Creative Commons Attribution-NonCommercial 3.0 Unported License which allows users to read, copy, distribute and make derivative works for non-commercial purposes from the material, as long as the author of the original work is cited properly. 